\title{
Different degeneration patterns of paraspinal muscles in degenerative lumbar diseases: a MRI analysis of 154 patients
}

\author{
Jun-zhe Ding ${ }^{1} \cdot$ Chao Kong $^{1} \cdot$ Xiang-yu Li ${ }^{1} \cdot$ Xiang-yao Sun $^{1} \cdot$ Shi-bao Lu ${ }^{1} \cdot$ Guo-gunag Zhao ${ }^{1}$
}

Received: 7 October 2020 / Revised: 20 October 2021 / Accepted: 7 November 2021 / Published online: 3 January 2022

(c) The Author(s) 2022

\begin{abstract}
Study design A retrospective study.

Objective To evaluate the different degeneration patterns of paraspinal muscles in degenerative lumbar diseases and their correlation with lumbar spine degeneration severity.

Summary of background data The degeneration characteristics of different paraspinal muscles in degenerative lumbar diseases remain unclear.

Methods 78 patients diagnosed with single-level degenerative lumbar spondylolisthesis (DLS) and 76 patients with degenerative lumbar kyphosis (DLK) were included as DLS and DLK groups. Paraspinal muscle parameters of psoas major (PS), erector spinae (ES) and multifidus muscle (MF) were measured, including fatty infiltration (FI) and relative cross-sectional area (rCSA), namely the ratio of the paraspinal muscle CSA to the CSA of the vertebrae of the same segment. Sagittal parameters including lumbar lordosis (LL) and sagittal vertical axis (SVA) were measured. The paraspinal muscle parameters and ES/MF rCSA ratio were compared between the two groups. Paraspinal muscles parameters including rCSA and FI were also compared between each segments from L1 to L5 in both DLS and DLK groups. In order to determine the influence of sagittal spinal alignment on paraspinal muscle parameters, correlation analysis was conducted between the MF, ES, PS rCSA and FI and the LL in DLS and DLK group.

Result MF atrophy is more significant in DLS patients compared with DLK. Also, MF fatty infiltration in the lower lumbar spine of DLS patients was greater compared to DLK patients. DLK patients showed more significant atrophy of ES and heavier ES fatty infiltration. MF FI was significantly different between all adjacent segments in both DLS and DLK groups. In DLS group, ES FI was significantly different between L2/L3 to L3/L4 and L4/L5 to L5/S1, while in DLK group, the difference of ES FI between all adjacent segments was not significant, and ES FI was found negatively correlated with LL. Conclusions Paraspinal muscles show different degeneration patterns in degenerative lumbar diseases. MF degeneration is segmental in both DLS and DLK patients, while ES degenerated diffusely in DLK patients and correlated with the severity of kyphosis. MF degeneration is more significant in the DLS group, while ES degeneration is more significant in DLK patients. MF is the stabilizer of the lumbar spine segments, while the ES tends to maintain the spinal sagittal balance.
\end{abstract}

Keywords Paraspinal muscle $\cdot$ Degenerative lumbar spondylolisthesis $\cdot$ Degenerative lumbar kyphosis $\cdot$ Relative crosssectional area $\cdot$ Fatty infiltration

Shi-bao Lu

445119837@qq.com

Guo-gunag Zhao

xuanwuspine2021@163.com

Jun-zhe Ding

18310693576@163.com

Chao Kong

2979022559@qq.com
Xiang-yu Li

1xyyx1@sina.com

Xiang-yao Sun

sun.xiang.yao@163.com

1 Department of Orthopedic, National Clinical Research Center for Geriatric Diseases, Xuanwu Hospital of Capital Medical University, No.45 Changchun Street, Beijing 100053, China 


\section{Introduction}

The degenerative change of paraspinal muscles has been proven to be associated with a various of lumbar disorders and diseases [1-3]. Paraspinal muscle is composed of the iliopsoas, quadratus lumborum, erector spinae (ES) and multifidus muscle (MF) $[4,5]$. The lumbar paraspinal muscle plays an irreplaceable role in the stability of spine and the maintenance of the coronal and sagittal alignment [6]. Also, the degree of muscle degeneration is associated with the spine degeneration and sagittal balance [7]. However, the anatomic characteristics, innervation and biomechanical effects of various muscles such as MF, ES and psoas major (PS) are not consistent. The anatomical location of the MF and the ES are close, and even the boundary between the two is difficult to distinguish on imaging. However, these two muscles are with different fascicles and innervation patterns [8]. MF fibers are orientated obliquely relative to the spine, while the erector spinae muscle bundle is substantially parallel to the body long axis. The cross-sectional area (CSA) of PS and MF in asymptomatic population increases caudalward gradually, while the CSA of the ES gradually decreases [3]. The role of paraspinal muscles in degenerative lumbar diseases has been recognized by a series of studies, indicating that paraspinal muscles are essential for lumbar spine motion and stabilization [6, 9-11]. MF is one of the most studied paraspinal muscles. Multiple studies have shown that MF is associated with various degenerative lumbar diseases such as low back pain, degenerative lumbar spondylolisthesis (DLS) and degenerative lumbar kyphosis (DLK) [12-14]. However, different degrees of degeneration in MF, ES and PS can often be observed in different patient populations. Whether the degenerative changes of the paraspinal muscles are related to the severity of spinal degenerative diseases remains controversial. It needs to be clear whether MF is the protagonist of a series of paraspinal muscles, and whether the different paraspinal muscles play the same role in degenerative lumbar diseases and exhibit consistent pattern of degeneration. Therefore, the present study focused on comparing the parameters of PS, ES and MF in patients with DLS and DLK, and to observe their correlation with disease severity. We hypothesizes that (1) patients with DLS and those with DLK will differ in the extent and segmental distribution of degenerative changes in MF, ES and PS muscles; (2) the degree of paraspinal muscles degeneration is related to the severity of the spinal disorder.

\section{Materials and methods}

\section{Patients}

The patient hospitalization number was used to encode demographic information of the subjects. All parties are fully aware of the confidentiality of the Helsinki Declaration. This study has been approved by the Center's Medical Ethics Committee.

Our retrospective study included 154 patients with degenerative lumbar disease diagnosed and treated at our center between November 2017 and June 2019. The average duration of symptom (pain) in patients was 11 months (1-36 months). Patients under 18 and over 90 years of age were excluded from the study. 78 patients diagnosed with single-level DLS and 76 patients with DLK were selected as subjects. Exclusion criteria included neuromuscular disease, spinal infections, ankylosing spondylitis, scoliosis, lumbar spinal tumor and patients with previous lumbar spine trauma or surgery.

DLS patients were diagnosed by three spinal surgeons based on subjective symptoms, clinical examinations and radiological data including $X$-ray and magnetic resonance image (MRI) [15]. DLK patients were diagnosed by three spinal surgeons based on the following clinical and radiographic features: (1) a forward stoop with difficulty walking due to axial back pain, (2) adaptive postural changes in an attempt to maintain a normal standing position, (3) loss of lumbar lordosis (LL) and (4) abnormal sagittal vertical axis (SVA). Radiographic evaluations of patients with suspected DLK were performed using a full-length 36-inch standing lateral radiograph of the entire spine [16]. Patients who are diagnosed with both DLS and DLK were excluded.

\section{Imaging technique}

In all patients, standing radiographs of the whole spine in the posteroanterior and lateral plane (Siemens DR system, Siemens, Germany) had been taken as part of their earlier clinical investigation. For the lateral films, the patients stood with their knees fully extended, the feet shoulderwidth apart, looking straight ahead, with their elbows bent and knuckles in the supraclavicular fossa bilaterally.

The MRI data were acquired on the Siemens Trio Tim 3.0 T MR imager (Siemens, Germany). The patients were placed in the supine position, with their legs straight and the lumbar spine in a neutral posture. Measurements were performed from the L1 superior endplate to the S1 superior endplate. Slices were obtained parallel to the superior endplate of the lower vertebra at each level. 


\section{Radiological parameters}

The radiological data of all patients were collected and measured by picture archiving and communication system (PACS). Measurement parameters include paraspinal muscle CSA, paraspinal muscle fatty infiltration (FI), LL and SVA. The muscle parameters (CSA and FI) were made on the middle layer of the MRI at each segment.

Paraspinal muscle CSAs were measured using the grayscale discrimination method proposed by Ranson et al. [17]. CSA was obtained by dividing the Region of interest (ROI) according to the boundaries of each paraspinal muscle on the cross section. Relative cross-sectional area (rCSA) was adopted to eliminate the individual differences in muscle volume that affect the results [18]. rCSA is the ratio of the paraspinal muscle CSA to the CSA of the vertebrae of the same segment. The mean value of both sides was measured and adopted for analysis.

To measure the degree of paraspinal muscles FI, we used the Image J Threshold method (National Institutes of Health,
Bethesda, MD, USA). Namely the percentage of the number of fat pixels in the total number of pixels in each paraspinal muscle ROI [19]. A threshold grayscale value of 120 was used to distinguish the pixels of intramuscular fatty tissue [20]. The measurement method is shown in Fig. 1.

The LL and SVA were measured on lateral radiographs. The LL is defined as the angle between the L1 vertebral superior endplate and the $\mathrm{S} 1$ vertebral superior endplate. The SVA was measured as the perpendicular distance between the $\mathrm{C} 7$ plumb line and the superior posterior corner of the S1 vertebra.

All parameters were measured by two orthopedic surgeons receive radiological training, respectively. Mean value of the two measurements was adopted for analysis.

\section{Statistical analysis}

Statistical analysis was conducted with SPSS 22.0 (IBM Corp., USA). Student $t$ test was conducted for comparison of FI, rCSA and sagittal radiological parameters between

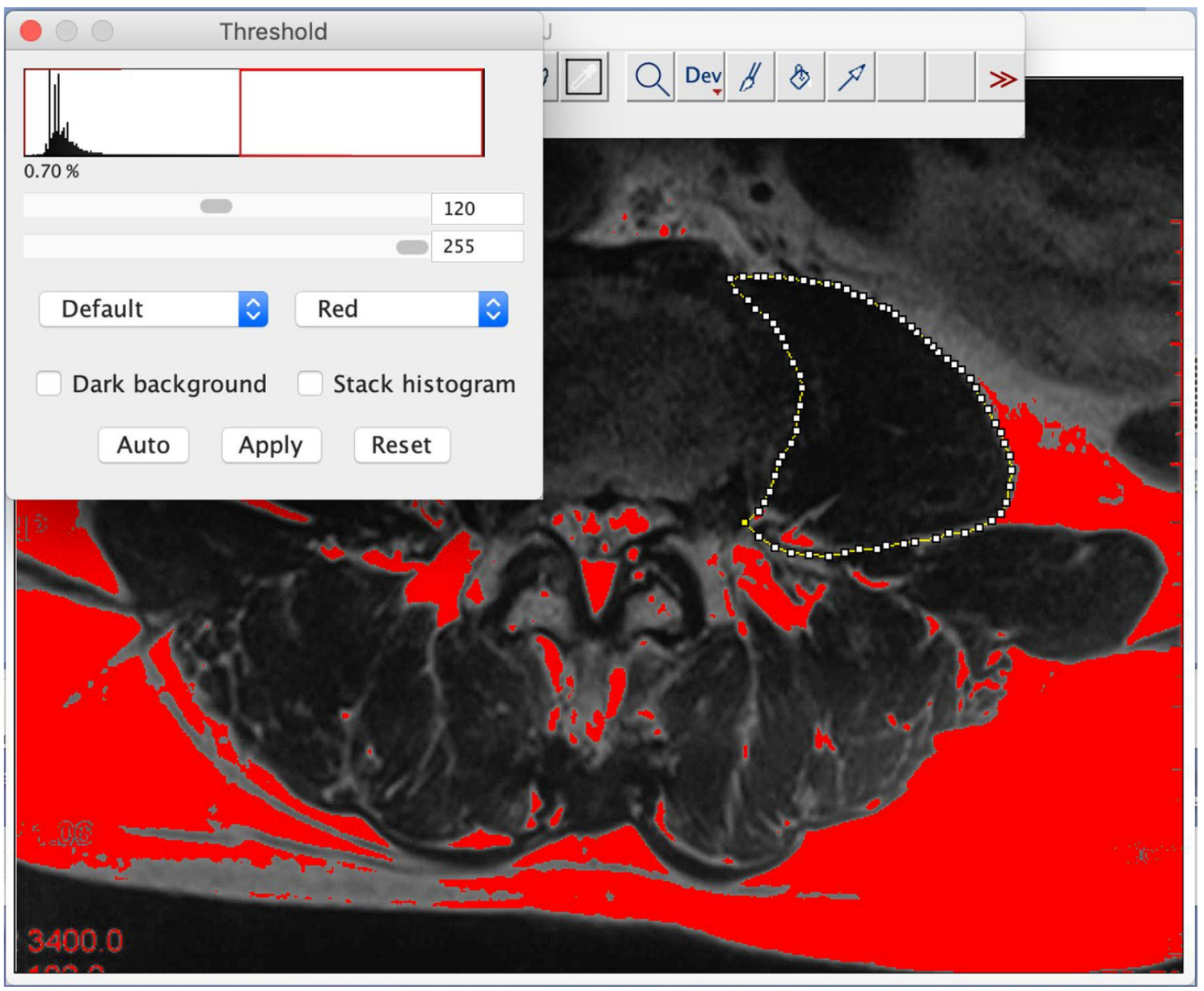

Fig. 1 Measurement method of the PS FI. The Image J Threshold method on the middle layer of magnetic resonance image in each segment. Red pixels represent fat tissues. PS psoas major, FI fatty infiltration 
two groups. The comparison of paraspinal muscle parameters between segments used mixed ANOVA with segmental level as the within-subject factor and the patient group as the between-subject factor. Post hoc comparisons were performed using a pairwise comparison with Bonferroni correction to examine the subgroup differences. The correlation between paraspinal muscle parameters and sagittal radiological parameters was analyzed by Pearson correlation test. Intraobserver reliability and the interobserver reliability for rCSA of PS, ES and MF were evaluated using the intraclass correlation coefficient (ICC) [21]. The data are presented as mean values \pm standard deviation. $P<0.05$ was considered to be statistically significant.

\section{Result}

\section{Patient demographics and radiological parameters}

The DLS group included 51 females and 27 males, with $69.2 \pm 8.5(52-84)$ years of age; the average body mass index (BMI) was $25.23 \pm 3.71$. The DLK group included 48 females and 28 males, with $70.1 \pm 7.3$ (56-83) years of age; the average BMI was $24.45 \pm 3.09$. Patients in the DLK group were older, but with no statistically significance $(p=0.482)$. Gender $(p=0.773, t=0.083)$ and BMI did not differ significantly between the two groups $(p=0.162)$. The most common segment of DLS was L4-L5. LL was $52.4 \pm 18.4^{\circ}$, SVA was $4.20 \pm 2.61 \mathrm{~mm}$, and slippage percentage was $33.7 \pm 10.6 \%(18.6-54.7 \%)$ in DLS group. In DLK group, LL was $6.8 \pm 9.7^{\circ}$ and SVA was $14.95 \pm 8.79 \mathrm{~mm}$. The LL in DLS group was significantly larger, and SVA was significantly smaller than that in the DLK group. Patient demographics and sagittal radiological parameters are summarized in Table 1.

\section{Paraspinal muscles parameters}

The results indicate that MF rCSA in DLS group was smaller than that in DLK group at all the segments from L1/L2-L5/S1, while MF FI in DLS group was higher than

Table 1 Demographics and sagittal radiological parameters of patients in DLS and DLK patients

\begin{tabular}{lccr}
\hline & DLS & DLK & $P$ value \\
\hline Age & $69.2 \pm 8.5$ & $70.1 \pm 7.3$ & $\mathbf{0 . 4 8 2}$ \\
BMI & $25.23 \pm 3.71$ & $24.45 \pm 3.09$ & $\mathbf{0 . 1 6 2}$ \\
SVA & $4.20 \pm 2.61$ & $14.95 \pm 8.79$ & $<\mathbf{0 . 0 0 1 *}$ \\
LL & $52.4 \pm 18.4$ & $6.8 \pm 9.7$ & $<\mathbf{0 . 0 0 1 *}$ \\
\hline
\end{tabular}

All values are expressed as mean value \pm standard deviation

$B M I$ Body mass index, SVA sagittal vertical axis, $L L$ lumbar lordosis that in DLK group at L4/L5 and L5/S1. ES rCSA in DLS group was larger than that in DLK group at L2/L3-L5/S1, and ES FI in DLS group was lower in L1/L2-L3/L4. There was no significant difference in PS rCSA and FI between the two groups. The paraspinal muscle rCSA and FI of DLS and DLK group are summarized in Table 2.

The comparison of ES/MF rCSA ratio between the two groups showed that the patients in the DLK group had smaller ratios in each segment of L1-L5 (Table 3).

In order to study the muscle degeneration segmentally, we compared the paraspinal muscles of each segment in both DLS and DLK groups. The rCSA and FI data for all muscles showed significant segmental differences, and for MF and ES, there were significant differences between patient groups. In addition, the FI of MF and ES has significant interaction effects between patient groups and lumbar segments (Table 4). It shows that the lumbar segments have different effects on the FI of MF and ES in the two groups. Result of post hoc comparisons showed MF FI was significantly different between all adjacent segments in both DLS and DLK group. In DLS group, ES FI was significantly different between L2/L3 to L3/L4 and L4/L5 to L5/S1, while in DLK group, the ES FI difference between all adjacent segments was not significant (Tables 5 and 6).

Intraobserver ICC was excellent for rCSA for PS, ES and MF [ICC $=0.994(95 \% \mathrm{CI}=0.982-0.997) ; \mathrm{ICC}=0.998(95 \%$ $\mathrm{CI}=0.996-0.999) ; \mathrm{ICC}=0.962(95 \% \mathrm{CI}=0.936-0.988)]$. The mean interobserver reliability was also good to excellent in measurement of the rCSA of PS, ES and MF in MRI. $[\mathrm{ICC}=0.972(95 \% \mathrm{CI}=0.966-0.986) ; \mathrm{ICC}=0.982(95 \%$ $0.964-0.992) ; \mathrm{ICC}=0.966(95 \% \mathrm{CI}=0.942-0.987)]$.

\section{Correlation analysis}

In order to determine the influence of sagittal spinal alignment on paraspinal muscle parameters, correlation analysis was conducted between both rCSA and FI of MF, ES and PS and the LL in DLS and DLK groups. In the DLK group, the ES FI in each segment was negatively correlated with LL (Fig. 2). Other parameters showed no significant correlation with LL.

\section{Discussion}

\section{Different degeneration patterns of paraspinal muscles}

The paraspinal muscles have been recognized as lumbar stabilizer and a protective factor in degenerative lumbar disease. Atrophy and fatty infiltration are the two major features of muscle degeneration $[22,23]$. Researches in the past two decades have shown that the degeneration and function of 
Table 2 Muscle degeneration of multifidus muscle, erector spinae and psoas major in DLS and DLK group

\begin{tabular}{|c|c|c|c|c|c|c|}
\hline & \multicolumn{3}{|l|}{$\mathrm{rCSA}$} & \multicolumn{3}{|l|}{ FI } \\
\hline & DLS & DLK & $P$ value & DLS & DLK & $P$ value \\
\hline \multicolumn{7}{|l|}{$M F$} \\
\hline $\mathrm{L} 1 / \mathrm{L} 2$ & $62.95 \pm 20.93$ & $72.60 \pm 25.95$ & $0.012 *$ & $27.73 \pm 3.70$ & $28.67 \pm 3.41$ & 0.104 \\
\hline L2/L3 & $82.77 \pm 33.86$ & $98.08 \pm 39.58$ & 0.011* & $36.37 \pm 4.64$ & $36.29 \pm 4.27$ & 0.911 \\
\hline $\mathrm{L} 3 / \mathrm{L} 4$ & $116.05 \pm 47.48$ & $137.43 \pm 55.09$ & 0.011* & $45.78 \pm 5.02$ & $46.71 \pm 5.29$ & 0.267 \\
\hline L4/L5 & $130.82 \pm 34.15$ & $149.72 \pm 38.39$ & $0.002 *$ & $61.08 \pm 5.69$ & $52.34 \pm 5.74$ & $<0.001 *$ \\
\hline $\mathrm{L} 5 / \mathrm{S} 1$ & $136.88 \pm 38.86$ & $152.24 \pm 40.58$ & 0.018* & $64.64 \pm 6.26$ & $55.54 \pm 5.97$ & $<0.001 *$ \\
\hline \multicolumn{7}{|l|}{$E S$} \\
\hline $\mathrm{L} 1 / \mathrm{L} 2$ & $113.33 \pm 60.11$ & $111.38 \pm 50.71$ & 0.828 & $24.82 \pm 8.43$ & $33.93 \pm 10.59$ & $<0.001 *$ \\
\hline L2/L3 & $118.12 \pm 41.56$ & $103.84 \pm 27.70$ & 0.013* & $27.36 \pm 9.67$ & $37.76 \pm 12.08$ & $<0.001 *$ \\
\hline L3/L4 & $102.94 \pm 31.95$ & $85.69 \pm 27.39$ & $<0.001 *$ & $36.32 \pm 8.55$ & $39.54 \pm 9.90$ & 0.032* \\
\hline L4/L5 & $90.35 \pm 29.35$ & $71.66 \pm 23.17$ & $<0.001 *$ & $38.31 \pm 10.52$ & $40.06 \pm 7.24$ & 0.231 \\
\hline $\mathrm{L} 5 / \mathrm{S} 1$ & $84.69 \pm 34.99$ & $65.64 \pm 28.09$ & $<0.001 *$ & $44.33 \pm 10.81$ & $42.62 \pm 7.63$ & 0.261 \\
\hline \multicolumn{7}{|l|}{$P S$} \\
\hline L1/L2 & $23.52 \pm 10.98$ & $25.62 \pm 13.46$ & 0.292 & $6.95 \pm 2.68$ & $6.76 \pm 2.25$ & 0.625 \\
\hline L2/L3 & $47.82 \pm 21.29$ & $55.32 \pm 27.21$ & 0.059 & $13.23 \pm 5.11$ & $12.96 \pm 5.32$ & 0.751 \\
\hline L3/L4 & $75.56 \pm 36.45$ & $85.97 \pm 42.46$ & 0.105 & $23.56 \pm 8.36$ & $23.40 \pm 9.10$ & 0.910 \\
\hline L4/L5 & $102.13 \pm 50.95$ & $114.46 \pm 59.40$ & 0.169 & $22.87 \pm 6.03$ & $21.54 \pm 6.40$ & 0.188 \\
\hline $\mathrm{L} 5 / \mathrm{S} 1$ & $101.98 \pm 51.52$ & $114.47 \pm 59.77$ & 0.166 & $25.67 \pm 6.92$ & $26.63 \pm 9.17$ & 0.466 \\
\hline
\end{tabular}

All values are expressed as mean value \pm standard deviation

$r C S A$ Relative paraspinal muscle cross area, FI fatty infiltration, $D L S$ degenerative lumbar spondylolisthesis, $D L K$ degenerative lumbar kyphosis, $M F$ multifidus muscle, $E S$ erector spinae, $P S$ psoas major
Table 3 Ratio of rCSA-ES/MF in DLS and DLK group

\begin{tabular}{lllr}
\hline rCSA-ES/MF & DLS & DLK & $P$ value \\
\hline L1/L2 & $1.84 \pm 0.77$ & $1.57 \pm 0.49$ & $\mathbf{0 . 0 1 2 *}$ \\
L2/L3 & $1.59 \pm 0.59$ & $1.21 \pm 0.51$ & $<\mathbf{0 . 0 0 1 *}$ \\
L3/L4 & $0.99 \pm 0.41$ & $0.71 \pm 0.34$ & $<\mathbf{0 . 0 0 1 *}$ \\
L4/L5 & $0.73 \pm 0.31$ & $0.50 \pm 0.20$ & $<\mathbf{0 . 0 0 1 *}$ \\
L5/S1 & $0.66 \pm 0.33$ & $0.46 \pm 0.25$ & $<\mathbf{0 . 0 0 1 *}$ \\
\hline
\end{tabular}

All values are expressed as mean value \pm standard deviation

$r C S A$ relative paraspinal muscle cross-sectional area, $D L S$ degenerative lumbar spondylolisthesis, $D L K$ degenerative lumbar kyphosis, $M F$ multifidus muscle, $E S$ erector spinae

Table 4 Muscles parameters comparison between lumbar segments and patient groups

\begin{tabular}{llccc}
\hline & & Patient group & Segments & Interaction effect \\
\hline MF & rCSA & $\mathbf{0 . 0 0 5}^{*}$ & $<\mathbf{0 . 0 0 1} *$ & $\mathbf{0 . 1 4 3}$ \\
& FI & $<\mathbf{0 . 0 0 1}^{*}$ & $<\mathbf{0 . 0 0 1 *}$ & $<\mathbf{0 . 0 0 1 *}$ \\
ES & rCSA & $\mathbf{0 . 0 0 2 *}$ & $<\mathbf{0 . 0 0 1 *}$ & $\mathbf{0 . 1 1 0}$ \\
& FI & $<\mathbf{0 . 0 0 1 *}$ & $<\mathbf{0 . 0 0 1 *}$ & $<\mathbf{0 . 0 0 1 *}$ \\
PS & rCSA & $\mathbf{0 . 1 3 0}$ & $<\mathbf{0 . 0 0 1 *}$ & $\mathbf{0 . 2 0 8}$ \\
& FI & $\mathbf{0 . 8 2 3}$ & $<\mathbf{0 . 0 0 1 *}$ & $\mathbf{0 . 0 7 9}$ \\
\hline
\end{tabular}

$r C S A$ Relative paraspinal muscle cross area, $F I$ fatty infiltration, $M F$ multifidus muscle, $E S$ erector spinae, $P S$ psoas major
Table 5 Post hoc comparisons of muscles degeneration between adjacent segments in DLS group

\begin{tabular}{|c|c|c|c|c|c|}
\hline & & $\begin{array}{l}\mathrm{L} 1 / \mathrm{L} 2-\mathrm{L} 2 / \\
\mathrm{L} 3\end{array}$ & $\begin{array}{l}\text { L2/L3-L3/ } \\
\text { L4 }\end{array}$ & $\begin{array}{l}\text { L3/L4-L4/ } \\
\text { L5 }\end{array}$ & L4/L5-L5/S1 \\
\hline \multirow[t]{2}{*}{ MF } & rCSA & $0.007 *$ & $<0.001 *$ & 0.110 & 1.000 \\
\hline & FI & $<0.001 *$ & $<0.001 *$ & $<0.001 *$ & $0.001 *$ \\
\hline \multirow[t]{2}{*}{ ES } & $\mathrm{rCSA}$ & 1.000 & 0.217 & 0.566 & 1.000 \\
\hline & FI & 1.000 & $<0.001 *$ & 1.000 & $0.001 *$ \\
\hline \multirow[t]{2}{*}{ PS } & $\mathrm{rCSA}$ & $0.001 *$ & $<0.001 *$ & $<0.001 *$ & 1.000 \\
\hline & FI & $<0.001^{*}$ & $<0.001 *$ & 1.000 & $0.045 *$ \\
\hline
\end{tabular}

$P$ values of the post hoc comparisons are shown in the table $r C S A$ Relative paraspinal muscle cross area, FI fatty infiltration;, $M F$ multifidus muscle, $E S$ erector spinae, $P S$ psoas major

paraspinal muscles are closely related to the process of lumbar degeneration and can affect the long-term clinical outcome of lumbar spine degenerative diseases [14, 16, 24-28]. Previous studies have indicated that in both DLS and DLK patients, MF and ES are significantly degenerated compared with healthy asymptomatic subjects [16, 29]. However, paraspinal muscle is a complex of iliopsoas, quadratus lumborum, ES and MF. Although these muscles are anatomical adjacent and radiographically contiguous, they are with different biomechanical properties and innervation. These 
Table 6 Post hoc comparisons of muscles degeneration between adjacent segments in DLK group

\begin{tabular}{|c|c|c|c|c|c|}
\hline & & $\begin{array}{l}\mathrm{L} 1 / \mathrm{L} 2-\mathrm{L} 2 / \\
\mathrm{L} 3\end{array}$ & $\begin{array}{l}\text { L2/L3-L3/ } \\
\text { L4 }\end{array}$ & $\begin{array}{l}\text { L3/L4-L4/ } \\
\text { L5 }\end{array}$ & L4/L5-L5/S1 \\
\hline \multirow[t]{2}{*}{$\mathrm{MF}$} & rCSA & $0.001 *$ & $<0.001 *$ & 0.653 & 1.000 \\
\hline & FI & $<0.001 *$ & $<0.001 *$ & $<0.001 *$ & $0.001 *$ \\
\hline \multirow[t]{2}{*}{ ES } & $\mathrm{rCSA}$ & 1.000 & $0.007 *$ & 0.090 & 1.000 \\
\hline & FI & 0.152 & 1.000 & 1.000 & 1.000 \\
\hline \multirow[t]{2}{*}{ PS } & $\mathrm{rCSA}$ & $<0.001 *$ & $<0.001 *$ & 0.001* & 1.000 \\
\hline & FI & $<0.001 *$ & $<0.001 *$ & 0.999 & $<0.001 *$ \\
\hline
\end{tabular}

$P$ values of the post hoc comparisons are shown in the table $r C S A$ Relative paraspinal muscle cross area, $F I$ fatty infiltration, $M F$ multifidus muscle, $E S$ erector spinae, $P S$ psoas major

muscles play different roles in the lumbar spine motion and exhibit different degenerative processes. At the same time, different degenerative lumbar diseases have different biomechanical changes. As two commonly seen degenerative diseases of the lumbar spine, the biomechanical changes of DLS and DLK are discriminative. DLS manifests as segmental lumbar instability, local slippage and increased LL $[30,31]$, while DLK presents a loss of physiological curvature of lumbar spine. This difference in biomechanical changes has led to different paraspinal muscles degeneration patterns.

\section{Degeneration patterns of MF and ES in degenerative lumbar diseases}

The present study showed MF atrophy is more significant in DLS patients compared with DLK, and DLS patients showed heavier MF fatty infiltration in the lower lumbar spine when compared to DLK patients. DLK patients showed more significant atrophy of ES at L2/L3 to L5/S1 segments, and heavier ES fatty infiltration at L1/L2 to L3/ 14 segments when compared to DLS patients. Furthermore, ES and MF showed different patterns of degeneration. In both DLS and DLK patients, MF fatty infiltration is segmental and increased in the lower lumbar spine. However, there is no segmental difference in the level of ES fatty infiltration in DLK patients, which is manifested as diffuse degeneration. The present study confirmed and extended the previous results. Xia et al. conducted a study on 32 patients with degenerative spinal kyphosis, and they measured rCSA of MF, ES and PS, and spinal-pelvic parameters including $\mathrm{C} 7$ sagittal vertical axis (SVA), thoracic kyphosis (TK), thoracolumbar kyphosis (TLK), LL, pelvic incidence (PI), sacral slope (SS) and pelvic tilt in DSK patients. The results showed the MF rCSA correlated with SVA, TK, LL and TLK, while the ES rCSA correlated with PI and SS, which indicated that MF and ES have different roles in maintaining the sagittal alignment of the spine, and there is no correlation between the PS and sagittal parameters [32]. Thakar et al. showed that segmental MF atrophy can be seen in patients with DLS; the ES may appear hypertrophic, which may be a compensation of the paraspinal muscles $[29,33]$. These results, along with the findings of the present study, may indicate that MF acts as a stabilizer of the lumbar spine segments, while the ES maintains the spinal sagittal balance.

\section{Factors of degeneration patterns for MF and ES}

The unique anatomical and biomechanical characteristics may have led to different degeneration patterns. MF is the largest and most medial of the deep lumbar paraspinal muscles, originates from the sacrum and the ilium and inserts into the spinous processes and laminae of the lumbar vertebrae in a fanning pattern. MF distributed segmentally and exert independent biomechanical effects in each segment. Also, MF is innervated by the medial branch of the dorsal rami uni-segmentally, each band of multifidus muscle receiving its innervation from one dorsal ramus only [34]. These independent anatomical features make MF tend to develop segmental degeneration. In DLS patients, due to intervertebral disc and facet joint degeneration, the segmental stability is compromised. As the stabilizer of the lumbar spine, MF bears a greater load on the segment, where slippage occurs which may lead to MF degeneration. On the other hand, lower MF function including innate weaker MF and segmental MF degeneration could also result in lumbar instability, accelerating the progression of DLS.

The ES of DLK patients exhibited multi-segmental degeneration, which is different from the segmental degeneration of MF. The MF degeneration of the present study was more significant compared to the healthy controls of previous reports. Niemeläinen et al. found the FI of MF in healthy controls was between 17 and $28 \%$ from L3 to $\mathrm{S} 1$, which is smaller than the MF FI in the present study (27-64\%) [35]. Also, Dorien et al. measured the parameters of lumbar muscles in patients with LBP and found MF FI was between 5 and $17 \%$ [36]. Lee et al. measured FI of the muscle compartment through L1-L4 levels in healthy controls with a mean value of $15.9 \%$, which is lower than both the MF FI and ES FI in the present study [19]. As a major force in maintaining spinal sagittal alignment, the ES loading increases segmentally in DLK because of the reduced muscle lever arms, resulting in severe degeneration [11]. Lumbar ES has a medial and lateral division, namely the longissimus and iliocostalis. Contrary to the MF, ES innervation shows intersegmental collateralization via intersegmental communicating loops [8, 37]. These 
Fig. 2 Correlation between Lumbar lordosis (LL) and Erector spinae fatty infiltration (ES FI) for each segment in the degenerative lumbar kyphosis (DLK) group
L1/L2

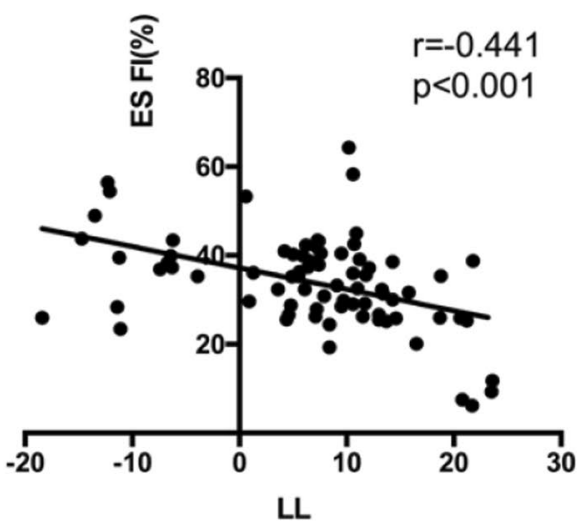

L3/L4

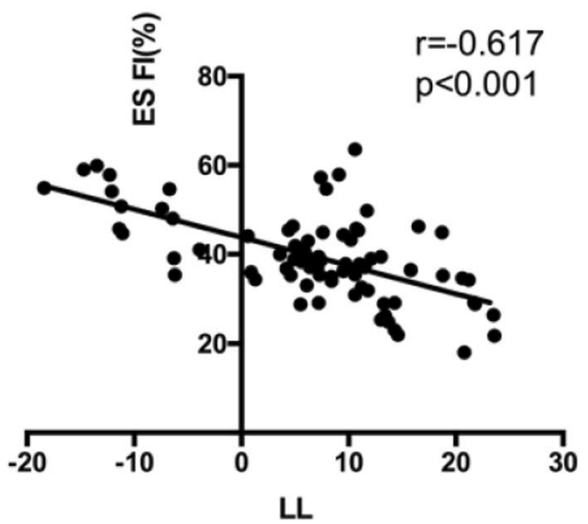

L2/L3

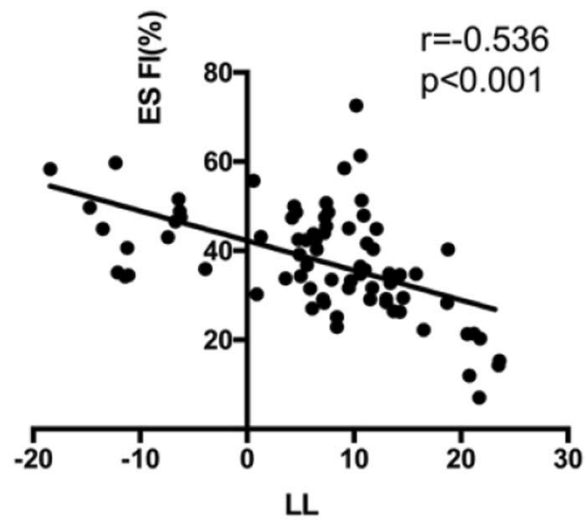

L4/L5

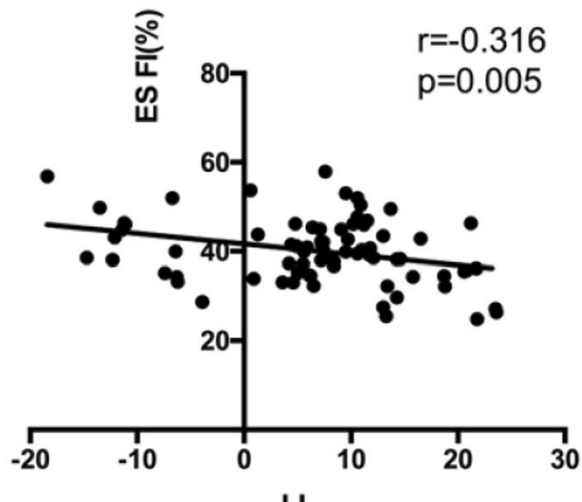

LL

\section{L5/S1}

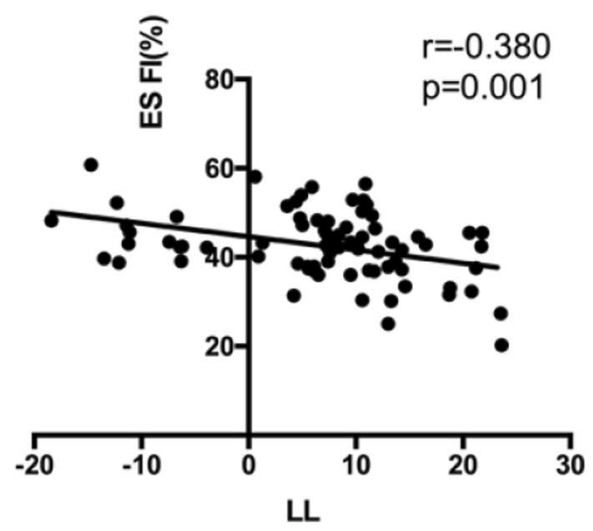

characteristics explain the multi-segmental ES degeneration in degenerative lumbar diseases.

\section{Paraspinal muscles degeneration in degenerative lumbar diseases}

MF and ES have different degeneration patterns in degenerative lumbar diseases. MF showed greater levels of fatty infiltration in the lower lumbar spine when compared to
ES. Moreover, the segmental degeneration is more significant in patients with DLS compared to those with DLK. In contrast, the ES degeneration is more significant in DLK patients. The severity of ES degeneration is correlated with the degree of kyphosis.

Paraspinal muscles are closely related to lumbar degeneration and affect the lumbar spine surgery outcome [38, 39]. Previous studies have shown that MF is important in maintaining lumbar lordosis [6]. Ineffectual muscular 
stabilization is one of the three major local reasons that probably lead to the development of DLS [40]. The traditional posterior approach dissects and seriously affects the paraspinal muscles, which interferes with postoperative recovery and normal spinal sequence maintenance. Studies have shown that patients with postoperative low back pain exhibit MF degeneration, including muscular atrophy, intramuscular adipose tissue accumulation and denervation [34]. The local anatomic structures make the ES and medial MF vulnerable to injury during traditional PLIF procedure [41]. The injury factors mainly include dissection, retraction, denervation and immobility. Procedures with less dissection and retraction, such as mini-invasive transforaminal lumbar interbody fusion can prevent the paraspinal muscles from injury $[42,43]$. Appropriate surgical approach and effective exercise can protect and strengthen the paraspinal muscles for potential positive impacts on clinical results, especially for the elderly patients [33].

\section{Conclusions}

Paraspinal muscles have different degeneration patterns in degenerative lumbar diseases. MF degeneration is segmental in both DLS and DLK patients, while ES degenerated diffusely in DLK patients and correlated with the severity of kyphosis. MF degeneration is more significant in the DLS group, while ES degeneration is more significant in DLK patients. MF is the stabilizer of the lumbar spine segments, while the ES tends to maintain the spinal sagittal balance. Appropriate surgical approach can be adopted to protect the paraspinal muscles for potential positive impacts on clinical results.

Funding This subject was funded by Beijing Municipal Medical Science Institute-Public Welfare Development Reform Pilot Project: (Capital Medical Research No. 2019-2).

Data availability The datasets used or analyzed during the current study are available from the corresponding author on reasonable request.

\section{Declarations}

Conflict of interest There are no conflicts of interest for the current study.

Ethics approval This study has been approved by the Center's Medical Ethics Committee.

Consent to participate All parties participated are fully aware of the confidentiality of the Helsinki Declaration.
Consent for publication Written informed consent for publication was obtained from all participants.

Open Access This article is licensed under a Creative Commons Attribution 4.0 International License, which permits use, sharing, adaptation, distribution and reproduction in any medium or format, as long as you give appropriate credit to the original author(s) and the source, provide a link to the Creative Commons licence, and indicate if changes were made. The images or other third party material in this article are included in the article's Creative Commons licence, unless indicated otherwise in a credit line to the material. If material is not included in the article's Creative Commons licence and your intended use is not permitted by statutory regulation or exceeds the permitted use, you will need to obtain permission directly from the copyright holder. To view a copy of this licence, visit http://creativecommons.org/licenses/by/4.0/.

\section{References}

1. Fortin M, Lazáry À, Varga PP, McCall I, Battié MC (2016) Paraspinal muscle asymmetry and fat infiltration in patients with symptomatic disc herniation. Eur Spine J 25(5):1452-1459. https://doi.org/10.1007/s00586-016-4503-7

2. Abbas J, Slon V, May H, Peled N, Hershkovitz I, Hamoud K (2016) Paraspinal muscles density: a marker for degenerative lumbar spinal stenosis? BMC Musculoskelet Disord 17(1):422. https://doi.org/10.1186/s12891-016-1282-6

3. Ranger TA, Cicuttini FM, Jensen TS, Heritier S, Urquhart DM (2019) Paraspinal muscle cross-sectional area predicts low back disability but not pain intensity. Spine J 19(5):862-868. https:// doi.org/10.1016/j.spinee.2018.12.004

4. Bogduk NCL(2012). Clinical and radiological anatomy of the lumbar spine 5 th ed

5. Moore KL, Agur AMR \& Dalley AF, Lippincott Williams \& Wilkins(2014). Clinically Oriented Anatomy 7th ed.

6. Banno T, Yamato Y, Hasegawa T, Kobayashi S, Togawa D, Oe S, Mihara Y, Kurosu K, Yamamoto N, Matsuyama Y (2017) Assessment of the Cross-Sectional Areas of the Psoas Major and Multifidus Muscles in Patients With Adult Spinal Deformity: A Case-Control Study. Clin Spine Surg 30(7):E968-E973. https://doi.org/10.1097/BSD.0000000000000480

7. Jun HS, Kim JH, Ahn JH, Chang IB, Song JH, Kim TH, Park MS, Chan Kim Y, Kim SW, Oh JK, Yoon DH (2016) The Effect of Lumbar Spinal Muscle on Spinal Sagittal Alignment: Evaluating Muscle Quantity and Quality. Neurosurgery 79(6):847855. https://doi.org/10.1227/NEU.0000000000001269

8. Kottlors M, Glocker FX (2008) Polysegmental innervation of the medial paraspinal lumbar muscles. Eur Spine J 17(2):300 306. https://doi.org/10.1007/s00586-007-0529-1

9. Hiyama A, Katoh H, Sakai D, Tanaka M, Sato M, Watanabe M (2019) The correlation analysis between sagittal alignment and cross-sectional area of paraspinal muscle in patients with lumbar spinal stenosis and degenerative spondylolisthesis. BMC Musculoskelet Disord 20(1):352. https://doi.org/10.1186/ s12891-019-2733-7

10. Liu Y, Liu Y, Hai Y, Li G, Liu T, Wang Y (2020) Lumbar lordosis reduction and disc bulge may correlate with multifidus muscle fatty infiltration in patients with single-segment degenerative lumbar spinal stenosis. Clin Neurol Neurosurg 189:105629. https://doi.org/10.1016/j.clineuro.2019.105629

11. Masaki M, Ikezoe T, Fukumoto Y, Minami S, Aoyama J, Ibuki S, Kimura M, Ichihashi N (2016) Association of walking speed with sagittal spinal alignment, muscle thickness, and echo intensity of lumbar back muscles in middle-aged and elderly women. 
Aging Clin Exp Res 28(3):429-434. https://doi.org/10.1007/ s40520-015-0442-0

12. Park MS, Moon SH, Kim TH, Oh J, Lee SJ, Chang HG, Shin JH (2018) Paraspinal Muscles of Patients with Lumbar Diseases. J Neurol Surg 79(4):323-329

13. Yagi M, Kaneko S, Yato Y, Asazuma T (2017) Drop Body Syndrome: A Distinct Form of Adult Spinal Deformity. Spine 42(16):E969-E977. https://doi.org/10.1097/BRS.0000000000 002012

14. Kalichman L, Hodges P, Li L, Guermazi A, Hunter DJ (2010) Changes in paraspinal muscles and their association with low back pain and spinal degeneration: CT study. Eur Spine J 19(7):1136-1144. https://doi.org/10.1007/s00586-009-1257-5

15. Ailon T, Smith JS, Shaffrey CI, Lenke LG, Brodke D, Harrop JS, Fehlings M, Ames CP (2015) Degenerative Spinal Deformity. Neurosurgery 77(Suppl 4):S75-S91. https://doi.org/10. 1227/NEU.0000000000000938

16. Hyun SJ, Bae CW, Lee SH, Rhim SC (2016) Fatty Degeneration of the Paraspinal Muscle in Patients With Degenerative Lumbar Kyphosis: A New Evaluation Method of Quantitative Digital Analysis Using MRI and CT Scan. Clin Spine Surg 29(10):441447. https://doi.org/10.1097/BSD.0b013e3182aa28b0

17. Ranson CA, Burnett AF, Kerslake R, Batt ME, O'Sullivan PB (2006) An investigation into the use of MR imaging to determine the functional cross sectional area of lumbar paraspinal muscles. Eur Spine J 15(6):764-773. https://doi.org/10.1007/ s00586-005-0909-3

18. Urrutia J, Besa P, Lobos D, Campos M, Arrieta C, Andia M, Uribe $S$ (2018) Lumbar paraspinal muscle fat infiltration is independently associated with sex, age, and inter-vertebral disc degeneration in symptomatic patients. Skeletal Radiol 47(7):955-961. https://doi.org/10.1007/s00256-018-2880-1

19. Lee JC, Cha JG, Kim Y, Kim YI, Shin BJ (2008) Quantitative analysis of back muscle degeneration in the patients with the degenerative lumbar flat back using a digital image analysis: comparison with the normal controls. Spine 33(3):318-325. https:// doi.org/10.1097/BRS.0b013e318162458f

20. Kim H, Lee CK, Yeom JS, Lee JH, Cho JH, Shin SI, Lee HJ, Chang BS (2013) Asymmetry of the cross-sectional area of paravertebral and psoas muscle in patients with degenerative scoliosis. Eur Spine J 22(6):1332-1338. https://doi.org/10.1007/ s00586-013-2740-6

21. Chen RQ, Hosogane N, Watanabe K, Funao H, Okada E, Fujita N, Hikata T, Iwanami A, Tsuji T, Ishii K, Abe T, Toyama Y, Nakamura M, Matsumoto M (2016) Reliability Analysis of SpinoPelvic Parameters in Adult Spinal Deformity: A Comparison of Whole Spine and Pelvic Radiographs. Spine 41(4):320-327. https://doi.org/10.1097/BRS.0000000000001208

22. Schlaeger S, Inhuber S, Rohrmeier A, Dieckmeyer M, Freitag F, Klupp E, Weidlich D, Feuerriegel G, Kreuzpointner F, Schwirtz A, Rummeny EJ, Zimmer C, Kirschke JS, Karampinos DC, Baum $\mathrm{T}$ (2019) Association of paraspinal muscle water-fat MRI-based measurements with isometric strength measurements. Eur Radiol 29(2):599-608. https://doi.org/10.1007/s00330-018-5631-8

23. Honkanen T, Mäntysaari M, Leino T, Avela J, Kerttula L, Haapamäki V, Kyröläinen H (2019) Cross-sectional area of the paraspinal muscles and its association with muscle strength among fighter pilots: a 5-year follow-up. BMC Musculoskelet Disord 20(1):170. https://doi.org/10.1186/s12891-019-2551-y

24. Hides J, Gilmore C, Stanton W, Bohlscheid E (2008) Multifidus size and symmetry among chronic LBP and healthy asymptomatic subjects. Man Ther 13(1):43-49. https://doi.org/10.1016/j.math. 2006.07.017

25. Lee SW, Chan CK, Lam TS, Lam C, Lau NC, Lau RW, Chan ST (2006) Relationship between low back pain and lumbar multifidus size at different postures. Spine 31(19):2258-2262. https://doi.org/ 10.1097/01.brs.0000232807.76033.33

26. Kjaer P, Bendix T, Sorensen JS, Korsholm L, Leboeuf-Yde C (2007) Are MRI-defined fat infiltrations in the multifidus muscles associated with low back pain? BMC Med 5:2. https://doi.org/10. 1186/1741-7015-5-2

27. Battié MC, Niemelainen R, Gibbons LE, Dhillon S (2012) Is level- and side-specific multifidus asymmetry a marker for lumbar disc pathology? Spine J 12(10):932-939. https://doi.org/10. 1016/j.spinee.2012.08.020

28. Chen YY, Pao JL, Liaw CK, Hsu WL, Yang RS (2014) Image changes of paraspinal muscles and clinical correlations in patients with unilateral lumbar spinal stenosis. Eur Spine J 23(5):999_ 1006. https://doi.org/10.1007/s00586-013-3148-Z

29. Wang G, Karki SB, Xu S, Hu Z, Chen J, Zhou Z, Fan S (2015) Quantitative MRI and X-ray analysis of disc degeneration and paraspinal muscle changes in degenerative spondylolisthesis. J Back Musculoskelet Rehabil 28(2):277-285. https://doi.org/10. 3233/BMR-140515

30. Gille, O., Challier, V., Parent, H., Cavagna, R., Poignard, A., Faline, A., Fuentes, S., Ricart, O., Ferrero, E., Ould Slimane, M., $\&$ French Society of Spine Surgery (SFCR) (2014) Degenerative lumbar spondylolisthesis: cohort of 670 patients, and proposal of a new classification. Orthopaedics Traumatol Surg Res OTSR 100(6 Suppl):S311-S315. https://doi.org/10.1016/j.otsr.2014.07. 006

31. Schuller S, Charles YP, Steib JP (2011) Sagittal spinopelvic alignment and body mass index in patients with degenerative spondylolisthesis. Eur Spine J 20(5):713-719. https://doi.org/10.1007/ s00586-010-1640-2

32. Xia W, Fu H, Zhu Z, Liu C, Wang K, Xu S, Liu H (2019) Association between back muscle degeneration and spinal-pelvic parameters in patients with degenerative spinal kyphosis. BMC Musculoskelet Disord 20(1):454. https://doi.org/10.1186/ s12891-019-2837-0

33. Thakar S, Sivaraju L, Aryan S, Mohan D, Sai Kiran NA, Hegde AS (2016) Lumbar paraspinal muscle morphometry and its correlations with demographic and radiological factors in adult isthmic spondylolisthesis: a retrospective review of 120 surgically managed cases. J Neurosurg Spine 24(5):679-685. https://doi.org/10. 3171/2015.9.SPINE15705

34. Klinger N, Yilmaz E, Halalmeh DR, Tubbs RS, Moisi MD (2019) Reattachment of the Multifidus Tendon in Lumbar Surgery to Decrease Postoperative Back Pain: A Technical Note. Cureus 11(12):e6366. https://doi.org/10.7759/cureus.6366

35. Niemelainen R, Briand MM, Battie MC (2011) Substantial asymmetry in paraspinal muscle cross-sectional area in healthy adults questions its value as a marker of low back pain and pathology. Spine 36(25):2152-2157

36. Goubert D, De Pauw R, Meeus M, Willems T, Cagnie B, Schouppe S, Van Oosterwijck J, Dhondt E, Danneels L (2017) Lumbar muscle structure and function in chronic versus recurrent low back pain: a cross-sectional study. Spine J 17(9):1285-1296. https://doi.org/10.1016/j.spinee.2017.04.025

37. Kalimo H, Rantanen J, Viljanen T, Einola S (1989) Lumbar muscles: structure and function. Ann Med 21(5):353-359. https://doi. org/10.3109/07853898909149220

38. Kim JY, Ryu DS, Paik HK, Ahn SS, Kang MS, Kim KH, Park JY, Chin DK, Kim KS, Cho YE, Kuh SU (2016) Paraspinal muscle, facet joint, and disc problems: risk factors for adjacent segment degeneration after lumbar fusion. Spine J 16(7):867-875. https:// doi.org/10.1016/j.spinee.2016.03.010

39. Pennington Z, Cottrill E, Ahmed AK, Passias P, Protopsaltis T, Neuman B, Kebaish KM, Ehresman J, Westbroek EM, Goodwin ML, Sciubba DM (2019) Paraspinal muscle size as an independent 
risk factor for proximal junctional kyphosis in patients undergoing thoracolumbar fusion. J Neurosurg Spine 31(3):380-388. https:// doi.org/10.3171/2019.3.SPINE19108

40. Kalichman L, Hunter DJ (2008) Diagnosis and conservative management of degenerative lumbar spondylolisthesis. Eur Spine J 17(3):327-335. https://doi.org/10.1007/s00586-007-0543-3

41. Hu ZJ, Fang XQ, Fan SW (2014) Iatrogenic injury to the erector spinae during posterior lumbar spine surgery: underlying anatomical considerations, preventable root causes, and surgical tips and tricks. Eur J orthopaedic Surg Traumatol 24(2):127-135. https:// doi.org/10.1007/s00590-012-1167-9

42. Bresnahan LE, Smith JS, Ogden AT, Quinn S, Cybulski GR, Simonian N, Natarajan RN, Fessler RD, Fessler RG (2017) Assessment of Paraspinal Muscle Cross-sectional Area After Lumbar Decompression: Minimally Invasive Versus Open
Approaches. Clin Spine Surg 30(3):E162-E168. https://doi.org/ 10.1097/BSD.0000000000000038

43. Tsutsumimoto T, Shimogata M, Ohta H, Misawa H (2009) Miniopen versus conventional open posterior lumbar interbody fusion for the treatment of lumbar degenerative spondylolisthesis: comparison of paraspinal muscle damage and slip reduction. Spine 34(18):1923-1928. https://doi.org/10.1097/BRS.0b013e3181 a9d28e

Publisher's Note Springer Nature remains neutral with regard to jurisdictional claims in published maps and institutional affiliations. 